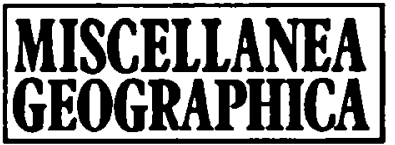

WARSZA WA 1996 Vol. 7

Ewelina Kantowicz

\title{
L'INTERDISCIPLINARITÉ DE LA PROTECTION DE L'ENVIRONNEMENT ET LES PROBLÈMES DE L'INTÉGRATION DES SCIENCES HUMAINES ET NATURELLES
}

L'organisation, dans certaines universités polonaises, d'études consacrées à la protection de l'environnement, a suscité depuis quelques années une discussion concernant leur programme et leurs bases théoriques et méthodologiques. L'approche pluri- ou interdisciplinaire est partout manifeste.

Il existe plusieurs manières de définir les notions de pluri- ou interdisciplinarité - aussi bien dans la recherche que dans l'enseignement. En ce qui concerne la recherche, la différence entre ces deux notions est souvent illustrée par deux situations extrêmes. Dans la première, les spécialistes de diverses disciplines se réunissent pour résoudre un problème, en apportant leurs connaissances, leurs méthodes et leurs points de vue. Dans la seconde, la résolution d'un problème exige de chacun de participants une parfaite connaissance de la conception de l'ensemble. La différence entre la pluridisciplinarité et l'interdisciplinarité réside donc dans le degré de l'intégration des chercheurs et des disciplines qu'ils représentent, autour du problème étudié.

En admettant cette signification de l'interdisciplinarité, il est question de savoir comment l'idée de l'intégration de diverses matières est réalisée dans le processus de l'enseignement: dans son programme, dans la composition de son contenu, dans la manière d'enseigner, dans la conception et les divers aspects de préparation des mémoires de maîtrise.

Nous allons aborder deux aspects seulement de ces problèmes, d'ailleurs les plus faciles à analyser: ceux concernant le programme d'enseignement et concernant la préparation des mémoires de maîtrise.

\section{L'INTERDISCIPLINARITÉ DE LA PROTECTION DE L'ENVIRONNEMENT ET LE PROGRAMME D'ENSEIGNEMENT}

Dix facultés participent, au sein de l'Université de Varsovie, dans la réalisation d'un programme d'études de protection de l'environnement. Ils 
représentent les disciplines différentes, aussi bien en ce qui concerne leur objet que les méthodes qu'elles utilisent. Selon la division dichotomique le plus souvent employée (Such 1987), ces disciplines peuvent être classées soit dans le groupe des sciences formelles (mathématiques), soit dans le groupe des sciences empiriques. Ces dernières se divisent ensuite, depuis les travaux de D. Hume au XVIII ${ }^{e}$ siècle, en sciences naturelles et humaines. Les disciplines enseignées au sein du programme peuvent donc être groupées de manière suivante (leurs noms correspondant aux appellations des différentes facultés à l'Université de Varsovie):

\begin{tabular}{|l|l|l|}
\hline \multirow{2}{*}{ Sciences formelles } & \multicolumn{2}{|c|}{ Sciences empiriques } \\
\cline { 2 - 3 } & \multicolumn{1}{|c|}{ naturelles } & \multicolumn{1}{c|}{ humaines } \\
\hline mathématique & biologie & philosophie \\
informatique & chimie & économie \\
mécanique & géographie & géographie \\
& physique & droit et administration \\
& géologie & sociologie \\
& & gestion \\
\hline
\end{tabular}

Ce schéma reflète la division des sciences institutionnalisées. Il nécessite un commentaire. Ainsi, la gestion, le droit et l'administration sont rarement classés dans le groupe des sciences empiriques humaines, puisqu'ils sont souvent considérés comme sciences appliqueées. Mais il s'agit là d'une autre division des disciplines scientifiques. Pour une autre raison, la géographie suscite, bien sûr, une réflexion. Elle figure à la fois dans le groupe des sciences naturelles et celui des sciences humaines. En effet, c'est une des disciplines limitrophes qui, de par la nature de l'objet de leurs études, font partie aussi bien des sciences naturelles que des sciences humaines. Tout comme, par exemple, la psychologie - qui est une science de l'homme, donc humaine, mais aussi biologique, donc naturelle. La géographie, comprise comme discipline qui traite "la différenciation de la surface de la Terre, les relations réciproques entre ses composants et la différenciation spatiale des activités des sociétés humaines" (Flis 1986, p. 11) peut être considérée comme science de la Terre, donc naturelle. Elle est aussi bien considérée comme science humaine - surtout, par exemple, en France, où elle fait partie des facultés des Lettres et où elle est enseignée en liaison avec l'histoire. Cette position limitrophe, propre à la géographie, suscite d'ailleurs constamment des discussions sur l'intégration des sciences naturelles et humaines ${ }^{1}$.

\footnotetext{
${ }^{1}$ Voir, entre autres, les textes de Johnston (1983) sur l'intégration de la géographie physique et humaine, de Kantowicz et Skotnicki (1984) sur l'intégration et désintégration de la géographie et de la géographie régionale.
} 
Analysons, du point de vue de cette intégration, le programme d'études de la protection de l'environnement, réalisé à l'Université de Varsovie. Il se compose, en gros, de quatre groupes de matières:

1. sciences mathématiques et naturelles (principes);

2. sciences humaines (principes) en $1^{\text {ère }}$ année - où cette intégration est donc la moins visible;

3. sciences du milieu naturel en $2^{\text {ème }}$ et $3^{\text {ème }}$ année. Les matières qui en font partie sont réunies en "troncs thématiques", se référant aux différentes "sphères": amosphère, lithosphère, hydrosphère, biosphère, étude du sol; s'y ajoutent les cours sur les techniques de recherche et les techniques de mesure. Le contenu de cet enseignement n'est donc pas celui qui correspond au terme anglo-saxon "environmental science"; ce dernier comprend en effet l'utilisation des sciences humaines et naturelles dans la résolution des "problèmes environnementaux" (de Groot 1992). Dans notre programme, autour des "troncs thématiques", une certaine intégration, au moins entre les différentes disciplines faisant partie des sciences naturelle, s'avère bien sûr nécessaire. Ainsi, par exemple, dans l'étude de l'hydrosphère, participent les hydrologues, les hydrogéologues, les hydrochimistes, les hydrobiologistes - représentant respectivement les facultés de Géographie, de Géologie et de Biologie. Cette intégration est également visible dans certaines matières, comme celle intitulée "Écologie avec éléments de toxycologie et la chimie analytique";

4. sciences appliquées, en $4^{\text {eme }}$ et $5^{\text {ème }}$ année (consacrées également à la préparation de mémoire de maîtrise), comprises dans le sens de l'utilisation des sciences naturelles et humaines dans le façonnement et la protection de l'environnement, ainsi que dans la politique écologique. Là, puisqu'il s'agit des problèmes pratiques, l'intégration est le mieux réussie. Presque chaque matière en est un exemple: Le milieu naturel comme base de la planification spatiale, Gestion du milieu naturel, Aspects juridiques de la protection de l'environnement. La nécessité de cette intégration apparaît également au cours de la préparation des mémoires de maîtrise; nous y avons affaire aux mêmes problèmes que ceux qui sont propres à la recherche.

\section{L'INTERDISCIPLINARITÉ DE LA PROTECTION DE L'ENVIRONNEMENT} ET LA PRÉPARATION DES MÉMOIRES DE MAÎTRISE

Il est d'abord nécessaire de préciser la nature de mémoire de maîtrise préparé dans le cadre des études de la protection de l'environnement qui ne disposent pas encore de bases théoriques suffisamment développées et cohérentes. On souligne, généralement, que ce mémoire doit être interdisciplinaire, doit présenter une appréciation (un projet) de l'état de l'environnement - et doit comporter une recherche concernant, le plus souvent, les causes de l'apparition des "problèmes environnementaux". 
Les sujets offerts aux étudiants, présentés par les différentes facultés, nécessitent soit une intégration au sein des sciences naturelles, soit au sein des sciences humaines (rarement), soit entre deux groupes de disciplines. Mais il s'agit non seulement d'une assimilation des étendues de diverses matières, mais aussi, ce qui est plus difficile, de l'intégration méthodologique. Pour mettre en ordre, de ce point de vue, les sujets des mémoires de maîtrise, essayons, suivant la démarche utilisée par Domański (1983), de voir à quels types de question doivent-ils répondre. Ces questions peuvent être les suivantes: 1. qu'est-ce? (intérrogation sur les faits), 2. comment est-ce? (interrogation sur la nature), 3 . de quoi cela dépend-il? (intérrogation sur les relations), 4. comment l'atteindre? (intérrogation sur l'optimalisation de la démarche). Voyons, suivant cette approche, quelques exemples de sujets de mémoires de maîtrise:

\begin{tabular}{|l|l|l|}
\hline \multicolumn{1}{|c|}{ Sujet } & \multicolumn{1}{|c|}{$\begin{array}{c}\text { Discipline } \\
\text { (Faculte) }\end{array}$} & \multicolumn{1}{c|}{ Type de question } \\
\hline $\begin{array}{l}\text { Les conditions naturelles et } \\
\text { économiques de l'utilisation des eaux } \\
\text { géothérmiques de la région } \\
\text { de Fodhale }\end{array}$ & $\begin{array}{l}\text { sciences naturelles } \\
\text { sciences humaines } \\
\text { (Fac. de Géologie) }\end{array}$ & $\begin{array}{l}\text { comment l'atteindre? } \\
\text { (optimalisation) }\end{array}$ \\
\hline $\begin{array}{l}\text { Appréciation des ressources } \\
\text { et des valeurs du milieu naturel } \\
\text { du point de vue des différents objectifs }\end{array}$ & $\begin{array}{l}\text { sciences naturelles } \\
\text { sciences humaines } \\
\text { (Fac. de Géographie, }\end{array}$ & $\begin{array}{l}\text { comment l'atteindre? } \\
\text { (optimalisation) }\end{array}$ \\
\hline $\begin{array}{l}\text { Caractéristique et réception } \\
\text { des données écologiques présentées } \\
\text { par les moyens de grande diffusion }\end{array}$ & $\begin{array}{l}\text { sciences humaines } \\
\text { (Fac. de Géographie) }\end{array}$ & $\begin{array}{l}\text { comment est-ce? } \\
\text { (nature) } \\
\text { qu'est-ce (faits) }\end{array}$ \\
\hline $\begin{array}{l}\text { Pollution des eaux souterraines } \\
\text { par les matières azotés; recherche } \\
\text { sur les méthodes de détermination }\end{array}$ & $\begin{array}{l}\text { sciences naturelles } \\
\text { (Fac. de Chimie) }\end{array}$ & $\begin{array}{l}\text { comment est-ce? } \\
\text { (nature) }\end{array}$ \\
\hline $\begin{array}{l}\text { Influence de la mosaíque du paysage } \\
\text { sur la différenciation des espèces } \\
\text { d'animaux }\end{array}$ & $\begin{array}{l}\text { sciences naturelles } \\
\text { (Fac. de Biologie) }\end{array}$ & $\begin{array}{l}\text { de quoi cela dépend-il? } \\
\text { (relation) }\end{array}$ \\
\hline $\begin{array}{l}\text { Diffusion de la pollution et inversion } \\
\text { de la température de l'air }\end{array}$ & $\begin{array}{l}\text { sciences naturelles } \\
\text { (Fac. de Géographie) }\end{array}$ & $\begin{array}{l}\text { de quoi cela dépend-il? } \\
\text { (relation) }\end{array}$ \\
\hline $\begin{array}{l}\text { Conditions naturelles et facteurs } \\
\text { de changements anthropogéniques } \\
\text { dans les résèrves de biosphère } \\
\text { UNESCO/MAB }\end{array}$ & $\begin{array}{l}\text { sciences naturelles } \\
\text { sciences humaines } \\
\text { (Fac. de Géographie, } \\
\text { Fac. de Biologie) }\end{array}$ & $\begin{array}{l}\text { comment est-ce? } \\
\text { (nature) } \\
\text { de quoi cela dépend-il? } \\
\text { (relations) }\end{array}$ \\
\hline $\begin{array}{l}\text { Conditions historiques et causes } \\
\text { des catastrophes dans la mine } \\
\text { de sel de Wieliczka }\end{array}$ & $\begin{array}{l}\text { sciences naturelles } \\
\text { sciences humaines } \\
\text { (Fac. de Géologie) }\end{array}$ & $\begin{array}{l}\text { de quoi cela dépend-il? } \\
\text { (relation) }\end{array}$ \\
\hline
\end{tabular}

Ainsi, tous les types de questions apparaissent dans les sujets de mémoires de maîtrise. Étant donné la complexité des processus qui s'opèrent dans le milieu naturel, la question concernant les relations s'avère la plus importante. En effet, dans la tradition la plus répandue d'explication scientifique, c'est-à-dire fondée sur les lois (le modèle nomologique et déductif) il s'agit 
de la question fondamentale: pourquoi? Mais elle n'a pas qu'une seule signification et, en fonction de la nature des lois, la réponse peut comporter (Siemianowski 1987):

- une explication fonctionnelle, concernant une relation fonctionnelle - ou une explication statistique, concernant une relation statistique (physique, chimie);

- une explication du suivi et de causalité, concernant les lois sur le développement, les lois historiques, du suivi dans le temps - et les lois de causalité (biologie, géologie, géographie, histoire, sociologie);

- une explication fonctionnelle où l'on demande "quel est le rôle", "quelle est la fonction" de l'objet étudié.

Ce rappel de la méthodologie générale démontre que dans le cadre d'un seul type de question, celle concernant les relations, il existe des différences quant à la nature de l'explication scientifique. Il faut prendre en compte ces différences lorsqu'on essaye d'intégrer les sciences naturelles et humaines. Car plusieurs difficultés peuvent apparaître. A commencer par celle qui découle de caractère particulier des sciences humaines, considérées souvent comme "sciences de compréhension", en opposition aux sciences naturelles, considérées comme "explicatives". Suivant ce point de vue, les sciences humaines, pour justifier une affirmation, n'utilisent pas les lois empiriques, mais les "actes spécifiques de connaissance", appelés "compréhension", par exemple la compréhension des motifs d'un comportement (Marciszewski 1987). Une autre différence entre les sciences naturelles et humaines est due au fait que ces dernières utilisent beaucoup plus souvent les critères du domaine d'axiologie - ceux du bien ou du mal, de beauté et de laideur, de conformité ou de non conformité aux intérêts du groupe. Donc, même si, dans la volonté d'une intégration, une conception générale sera admise (comme celle, par exemple, d'expliquer à l'aide des lois), les controverses persisteront. Le plus souvent on s'intérroge s'il est possible d'apporter des explications fondées uniquement sur les généralisations historiques - contrairement aux sciences naturelles, fondées sur les lois empiriques.

Mais revenons à nos mémoires de maîtrise. Comme on le voit, ils concernent aussi bien les aspects pratiques que théoriques. Ces derniers dominent, bien que nous sommes loin de considérer la protection de l'environnement comme une nouvelle discipline, distincte et indépendante. Plusieurs disciplines ont été créées aux confins des disciplines classiques et reconnues - comme la biochimie et, surtout, la cybernétique, utilisant aussi bien les sciences fondamentales que les sciences appliquées, mathématiques et empiriques, naturelles et humaines. Ces nouvelles disciplines jouent un rôle de plus en plus important. Mais le processus de leur création est complexe et ne résulte pas d'un simple cumul des travaux entrepris autour d'un nouveau champ de recherche. Dans le domaine de la protection de l'environnement nous assistons à une avalanche de publications. Certaines, de caractère interdisciplinaire, concernent le plus souvent 
les projets très concrets ou les politiques écologiques. D'autres, réalisées dans le cadre des différentes disciplines, sont en général sectorielles, rarement interdisciplinaires. Enfin, les publications de vulgarisation, ou relatives à l'éducation, sont très nombreuses. Tous ces travaux ne justifient pas, bien sûr, le caractère indépendent des études sur la protection de l'environnement. Dans cette situation, il ne reste qu'à chercher à atteindre une véritable interdisciplinarité, s'exprimant dans l'intégration des différentes matières.

\section{BIBLIOGRAPHIE}

Domański R., 1983, Teoretyczne podstawy geografii ekonomcznej (Fondements théoriques de la géographie économique), PWE, Warszawa.

Flis J., 1986, Szkolny stownik geograficzny (Dictionnaire scolaire de géographie), PWSiP, Warszawa.

Groot M. T. de, 1992, Environmental Science Theory, Leiden University.

Johnst on B. J., 1983, Resource analysis, resource management and integration of physical and human geography, Progress in Physical Geography, 7, 1, pp. 127-146.

Kant owicz E., Skotnicki M., 1984, Trends in regional geography, Miscellanea Geographica, pp. 153-161.

Marciszewski W., 1987, Nauka, in: Filozofia a nauka. Zarys encyklopedyczny (réd.: Z. Cackowski, J. Kmita, K. Szaniawski), Ossolineum, Wrocław (Science, in: La philosophie et la science. Esquisse encyclopédique).

S u ch J., 1987, Klasyfikacja nauk (Classification des sciences), ibid., pp. 297-305.

Si e mia now ski A., 1987, Wyjaśnienie naukowe (Explication scientifique), ibid., pp. 742-752. 